\title{
Multiple Signaling Pathways Are Involved in the Interleukine-4 Regulated Expression of DC-SIGN in THP-1 Cell Line
}

\author{
Changzhong Jin, ${ }^{1}$ Lijuan Wu, ${ }^{1,2}$ Jie Li, ${ }^{1,3}$ Meixin Fang, ${ }^{1}$ Linfang Cheng, ${ }^{1}$ and Nanping Wu ${ }^{1}$ \\ ${ }^{1}$ State Key Laboratory for Diagnosis and Treatment of Infectious Disease, The First Affiliated Hospital, School of Medicine, \\ Zhejiang University, Hangzhou 310003, China \\ ${ }^{2}$ Department of Internal Medicine, School of Medicine, Taizhou College, Taizhou 317000, China \\ ${ }^{3}$ Department of Infectious Disease, Second Affiliated Hospital of Wenzhou Medical College, Wenzhou 325027, China \\ Correspondence should be addressed to Nanping Wu, flwnp@yahoo.com.cn
}

Received 20 November 2011; Revised 8 February 2012; Accepted 8 February 2012

Academic Editor: Anthony L. DeVico

Copyright ( $\odot 2012$ Changzhong Jin et al. This is an open access article distributed under the Creative Commons Attribution License, which permits unrestricted use, distribution, and reproduction in any medium, provided the original work is properly cited.

\begin{abstract}
Dendritic cell-specific intercellular adhesion molecule-3 grabbing nonintegrin (DC-SIGN) is an important pattern recognition receptor on dendritic cells (DCs), and its expression shows significant cytological and histological specificity, being interleukine-4 (IL-4) dependent. The signaling pathways through which IL-4 regulates expression of DC-SIGN are still unclear. We used phorbol 12-myristate 13-acetate- (PMA-) differentiated THP-1 cells as the in vitro model of monocyte/macrophage cells to study the signaling pathways involved in IL-4-regulated expression of DC-SIGN. We found that a high expression of DC-SIGN could be induced by IL- 4 at the levels of mRNA and cell surface protein. Upregulated expression of DC-SIGN was almost completely blocked by the specific inhibitor of ERK pathway, and partly reduced by the specific inhibitors of JAK-STAT and NF- $\kappa$ B pathways. The activation of the three signaling pathways was directly confirmed by testing the phosphorylation of protein kinase within the cytoplasm and nucleus over time. The analysis of cis-acting elements of DC-SIGN promoter showed that the activity of DC-SIGN promoter without Ets-1 transcription factors binding site almost completely disappeared. Our results demonstrated that multiple signaling pathways are involved in IL-4 induced high expression of DC-SIGN on THP-1 cells, in which ERK pathway is the main signaling pathway and mediated by the Ets-1 transcription factors binding site.
\end{abstract}

\section{Introduction}

Dendritic cell-specific intercellular adhesion molecule (ICAM)-3-grabbing nonintegrin (DC-SIGN; also called CD209) is a type II transmembrane protein with a C-type lectin extracellular domain $[1,2]$. Functionally, DC-SIGN mediates antigen capture for processing and presentation in the context of major histocompatibility complex class II molecules $[3,4]$. DC-SIGN plays an important role in establishing the initial contact between dendritic cells (DCs) and resting T lymphocytes through its recognition of ICAM-3 [2] and also mediates DCs trafficking through interactions with endothelial ICAM-2 [5]. In addition, as a pattern recognition receptor (PRR), DC-SIGN function is subverted by numerous pathogens as a strategy to escape from immunosurveillance $[6,7]$. In the case of human immunodeficiency virus (HIV), DC-SIGN binds gp120 with high affinity [1], captures
HIV in the periphery, and promotes efficient infection of cells expressing HIV receptors and coreceptors $[8,9]$. DCSIGN also mediates virus uptake by dendritic cells through endocytosis, where HIV remains infectious for prolonged periods of time, evades immune surveillance, and is efficiently transferred to $\mathrm{CD} 4+\mathrm{T}$ cells in lymph nodes, like a "Trojan horse" [8]. Therefore, DC-SIGN plays a key role in HIV transmission, especially in terms of sexual transmission.

Expression of DC-SIGN is restricted to specific cells and tissues. DC-SIGN is preferentially expressed on immature DCs in peripheral tissues and in-vitro-derived monocytederived dendritic cells (MDDCs) $[2,5]$ and is usually considered as a DC-specific phenotypic marker. It has also been detected on synovial, placenta, and alveolar macrophages, and on a small subset of CD14+ peripheral blood DCs [10-12]. Trumpfheller et al. [13] analyzed the function of DC-SIGN in different cells, including monocyte-derived 
DCs, and three different DC-SIGN-expressing transfectants termed THP-1, 293, and HOS. They found that the function of DC-SIGN in HIV-1 transmission depends on its cellular context, since only DCs and the THP monocyte cell line, but not 293 and HOS, are able to use DC-SIGN to retain HIV1 in a highly infectious state for several days. The existing studies indicate that the expression and function of DCSIGN is precisely regulated. However, less is known about the mechanism of the regulated expression of DC-SIGN. Relloso et al. [14] found that the expression of DC-SIGN on MDDCs was induced by interleukine-4 (IL-4) through the JAK-STAT signaling pathway. However, the ERK signaling pathway is also considered to be a candidate pathway for DC-SIGN expression since it is involved in most IL-4/IL-4 receptor effects $[15,16]$. Given the important role of the functional activities displayed by DC-SIGN, it is necessary to determine the signaling pathways and factors controlling its expression.

Since the low level of DCs in human peripheral blood, immature MDDC in vitro studies is mostly derived from peripheral blood monocytes in the presence of GM-CSF and IL-4 [17, 18]. The expression of DC-SIGN is largely dependent on IL-4 [14], a cytokine whose action drives monocyte/macrophages into the "alternative activation" pathway [19]. THP-1 cells, which are widely used as a monocyte/macrophage differentiation model $[20,21]$, can be induced to express functional DC-SIGN when differentiated by protein kinase $\mathrm{C}$ (PKC) activators and IL-4 [22]. It has been reported that the signaling mechanisms regulating DC-SIGN expression in monocyte-derived macrophages and DCs also control the expression of this pathogen receptor in differentiated THP- 1 cells, implying that the THP- 1 cell line represents a useful cellular system for the analysis of the regulated expression and functional activities of DC-SIGN [14]. Here, we used THP- 1 cells as the in vitro model of MDDCs to study the signaling pathways of IL-4-induced expression of DC-SIGN. We found that multiple signaling pathways were involved in the process of IL-4-regulated DC-SIGN expression, the major of which was the ERK signaling pathway.

\section{Materials and Methods}

2.1. Cytokines and Antibodies. Recombined human IL-4 was obtained from R\&D System (Minneapolis, USA) and used at 1000 units/mL. PMA was obtained from Sigma (St. Louis, USA) and used at $10 \mathrm{ng} / \mathrm{mL}$. The NF- $\kappa$ B inhibitor Helenalin, JAK2/3 inhibitor AG490, p38MAPK inhibitor SB202190, and the MEK1/2 inhibitor PD98059 were purchased from Merck (Gibbstown, USA) and used at $1 \mu \mathrm{M}$ (Hellenalin), $300 \mu \mathrm{g} / \mathrm{mL}$ (AG490), $5 \mu \mathrm{M}$ (SB202190), and $50 \mu \mathrm{M}$ (PD98059). Mouse anti-human DC-SIGN monoclonal antibody was obtained from R\&D System.

2.2. Cell Culture. The acute monocytic leukemia cell line THP-1 was derived from the American Type Culture Collection (ATCC, Rockville, USA) and cultured in RPMI 1640 medium supplemented with $10 \%$ fetal bovine serum (complete medium), being maintained at $3-5 \times 10^{5}$ cells $/ \mathrm{mL}$. THP- 1 cells were routinely seeded at $5 \times 10^{5}$ cells $/ \mathrm{mL}$ in tissue culture dishes, and differentiation was induced by treatment with PMA $(10 \mathrm{ng} / \mathrm{mL})$ either alone or in combination with IL-4 (1000 units/mL). IL-4 was added into the cells after treatment with PMA for $18 \mathrm{~h}$ with complete medium and starvation for $6 \mathrm{~h}$ with RPMI 1640 medium supplemented with PMA $(10 \mathrm{ng} / \mathrm{mL})$. Fetal bovine serum was added to $10 \% 2 \mathrm{~h}$ after IL- 4 addition and differentiation was allowed to proceed for $72 \mathrm{~h}$. For inhibition of signaling pathways, inhibitors of the signaling pathways were added 30 min before IL- 4 addition and the process of differentiation was as per the above description. For subsequent analysis, differentiated THP-1 cells were detached from tissue culture plates by incubation in PBS $10 \mathrm{mM}$ EDTA on ice.

2.3. RNA Extraction and Real-Time Quantitative PCR. Total RNA from untreated or differentiated by PMA, PMA, plus IL-4, or signaling-inhibitor-treated THP-1 cells was extracted using total RNA extraction kit (Invitrogen) and reverse transcribed into cDNA in a total volume of $20 \mu \mathrm{L}$. Real-time polymerase chain reaction (PCR) of DC-SIGN mRNA was performed by amplifying samples of target cDNA in a DNA Engine Chromo 4 real-time quantitative PCR system (BioRad, Hercules, USA) using an SYBR Green kit (Roche Diagnostics $\mathrm{GmbH}$ ). Oligonucleotides were used for DC-SIGN mRNA amplification (CD209 sense: 5'-GGGAATTCAGAGTGGGGTGACATGAGTGAC-3' and CD209 antisense: $5^{\prime}$-CCCCAAGCTTGTGAAGTTCTGCTACGCAGGAG-3') and the inner conference $\beta$-actin mRNA amplification $(\beta$ actin sense: $5^{\prime}$-GGGAATTCAGAGTGGGGTGACATGAGTGAC-3' and $\beta$-actin antisense: $5^{\prime}$-CCCCAAGCTTGTGAAGTTCTGCTACGCAGGAG-3'). The reaction system was a mixture of $10 \mu \mathrm{L}$ SYBR Green Master Mix (Qiagen), $0.2 \mu \mathrm{L}$ of each oligonucleotides primers, $2 \mu \mathrm{L} \mathrm{cDNA}$, and the final volume was taken to $20 \mu \mathrm{L}$ with water. Real-time PCR was performed for 40 cycles of denaturation $\left(95^{\circ} \mathrm{C}\right.$, $45 \mathrm{~s})$, annealing $\left(62^{\circ} \mathrm{C}, 30 \mathrm{~s}\right)$, and extension $\left(72^{\circ} \mathrm{C}, 30 \mathrm{~s}\right)$, and double-stranded DNA was measured at $86^{\circ} \mathrm{C}$ after each cycle. Each sample was tested three times.

2.4. Flow Cytometry and Antibodies. Expression of DC-SIGN on surface of untreated or differentiated by PMA, PMA+IL4 , or signaling-inhibitor-treated THP-1 cells was determined by flow cytometry. The harvested THP-1 cells were washed twice with PBS, incubated with $10 \mu \mathrm{L}$ mouse anti-human DC-SIGN monoclonal antibody $(25-50 \mu \mathrm{g} / \mathrm{mL})$ for $1 \mathrm{~h}$, and washed again. Secondary antibody was added and incubated for $1 \mathrm{~h}$. Flow cytometry analysis was performed with a fourcolor FACScan flow cytometer (Becton Dickinson). Results are expressed as the expression index: percentage of markerpositive cells and the MFI.

2.5. Detection of Signaling Kinases and Factors in Cytoplasm and Nucleus of THP-1 Cells. THP-1 cells differentiated with PMA for $18 \mathrm{~h}$ were starved for $6 \mathrm{~h}$ with RPMI 1640 medium supplemented with PMA $(10 \mathrm{ng} / \mathrm{mL})$ and then induced with IL-4 (1000 units $/ \mathrm{mL})$. Cells were put on ice and washed by ice cooled PBS to stop the phosphorylation of the kinases and factors of signaling pathways $0 \mathrm{~min}, 5 \mathrm{~min}, 10 \mathrm{~min}$, 
TABLE 1: The sequence of the primers for DC-SIGN, the promoters, and internal reference.

\begin{tabular}{lc}
\hline & Sequence of the primers \\
\hline P1 (the forward primer of DC-SIGN promoter) & $5^{\prime}$-ATCATACGCGTATGAGTCCTTCTCCCTGTC-3' \\
P2 (the reverse primer of DC-SIGN promoter) & $5^{\prime}$-TGTAAGATCTGTCACCCCACTCTCCCCCAG-3' \\
P3 (5' side primer of AP-1 site) & $5^{\prime}$-CAAAAGACTAGTGGAAAGCATCAGA-3' \\
P4 (3' side primer of AP-1 site) & $5^{\prime}$-CCCTACCCAACTTCCTGTTTCTCTT-3' \\
P5 (joint primer beside AP-1 site) & $5^{\prime}$-CCACTAGTCTTTTGCCCTACCCAACTT-3' \\
P6 (5'side primer of Ets-1 site) & $5^{\prime}$-TGGGTAGGGATCTGTCATCCA-3' \\
P7 (3' side primer of Ets-1 site) & $5^{\prime}$-TCTCTTTCTGTGGGAGACTAGAT-3' \\
P8 (joint primer beside Ets-1 site) & $5^{\prime}$-CCACAGAAAGAGATGGGTAGGGATCT-3' \\
P9 (the forward primer of DC-SIGN) & $5^{\prime}$-AGAGTGGGGTGACATGAGTGAC-3' \\
P10 (the reverse primer of DC-SIGN) & $5^{\prime}$-GTGAAGTTCTGCTACGCAGGAG-3' \\
P11 (the forward primer of $\beta$-Actin) & $5^{\prime}$-CTCCATCCTGGCCTCGCTGT-3' \\
P12 (the reverse primer of $\beta$-Actin) & $5^{\prime}$-GCTGTCACCTTCACCGTTCC-3' \\
\hline
\end{tabular}

ACGCGT: the restriction sites of MLu I; AGATCT: the restriction sites of Bgl II.

$20 \mathrm{~min}, 30 \mathrm{~min}$, and $60 \mathrm{~min}$ after the addition of IL-4. Cytoplasmic protein and nuclear protein were extracted using cytoplasmic/nuclear protein extraction kit (PIERCE).

Phosphorylated and nonphosphorylated signaling kinases and factors were determined by Western Blot as described before [23]. $10 \mu \mathrm{g}$ of each sample was subjected to SDSPAGE under reducing conditions and transferred onto an immobilon polyvinylidene difluoride membrane (Millipore, Bedford, USA). After blocking with 5\% nonfat dry milk in $50 \mathrm{mM}$ Tris- $\mathrm{HCl}, \mathrm{pH} 7.6,150 \mathrm{mM} \mathrm{NaCl}, 0.1 \%$ Tween 20 , $1-2 \mu \mathrm{g} / \mathrm{mL}$ primary antibodies were added and incubated overnight at $4^{\circ} \mathrm{C}$. After incubation with HRP labeled secondary antibodies for $2 \mathrm{~h}$, the membrane was exposed with the VersaDoc 5000MP Image Analysis System (BioRad). Detection of signaling kinases and factors was carried out using specific monoclonal antibodies anti-STAT6, anti-ERK1/2, anti-NF- $\kappa$ Bp65, anti-I $\kappa \mathrm{B}$, and anti-p38. And phosphorylated kinases and factors were detected using anti-phospho-STAT6-Tyr641, anti-phospho-ERK1/2Thr202/Tyr204, anti-phospho-NF- $\kappa$ Bp65-Ser536, antiphospho-I $\kappa$ B-Thr19/Ser23, and anti-phospho-p38 MAPKThr180/Tyr182 (Cell Signaling Technology, Beverly, USA). $\beta$-actin and tubullin were detected as the inner reference using the polyclonal antibodies.

2.6. Construction of DC-SIGN Promoter Luciferase Reporter Plasmids and the Activity Detection. Total DNA was extracted from THP-1 cells and the whole region of DC-SIGN promoter was amplified by PRC using the forward and reverse primers of $\mathrm{P} 1$ and $\mathrm{P} 2$, where underlined residues represent additional sequences containing MLu I or Bgl II restriction sites, as shown in Table 1. The fragments of DC-SIGN promoter on both sides of AP- 1 were amplified using the forward and reverse primers of $\mathrm{P} 1$ and $\mathrm{P} 3$, and P2 and P4 separately, and then were linked by PRC with a combing primer $\mathrm{P} 5$, and forward and reverse primers of $\mathrm{P} 1$ and P2, conforming the DC-SIGN promoter without AP-1 binding site. And the DC-SIGN promoter without
Ets-1 binding site was amplified in the same way, using primers of $\mathrm{P} 1, \mathrm{P} 2, \mathrm{P} 6, \mathrm{P} 7$, and $\mathrm{P} 8$, as shown in Table 1. The mixture of PCR reaction consisted of $0.2 \mu \mathrm{L}$ DNA template, $2 \mu \mathrm{L}$ forward/reverse primers (10 mM), $4 \mu \mathrm{L} \mathrm{MgCl}_{2}(25 \mathrm{mM})$, $4 \mu \mathrm{L}$ dNTP $(2.5 \mathrm{mM}), 5 \mu \mathrm{L} 10 \times$ PCR buffer, $0.5 \mu \mathrm{L}$ Pfu DNA polymerase $(2 \mathrm{U} / \mu \mathrm{L}$, promega), and the final volume was taken to $50 \mu \mathrm{L}$ with water. PCR was performed for 30 cycles of denaturation $\left(95^{\circ} \mathrm{C}, 30 \mathrm{~s}\right)$, annealing $\left(57-62^{\circ} \mathrm{C}, 1 \mathrm{~min}\right)$, and extension $\left(72^{\circ} \mathrm{C}, 1 \mathrm{~min}\right)$. The fragments were identified by gene sequencing (Invitrogen). The fragments of DC-SIGN promoters were double digested with MLu I and Bgl II and gel purified and ligated into MLu I- and Bgl II-digested pGL3/Basic and pGL-3/Enhancer luciferase reporter vectors to generate complete DC-SIGN promoter luciferase reporter plasmids and those without AP-1 and Ets-1bingding sites. Transfection of DC-SIGN promoter luciferase reporter plasmids and the inner control pRL-TK in Hacat and 293T cells (ATCC) was accomplished using Trans Fast (Promega). DC-SIGN promoter luciferase reporter plasmids and the inner control pRL-TK were electransfected into THP-1 cells using Amaxa cell line transfection kit (Amaxa, Germany) in Amaxa Nucleofector electroporation apparatus (Amaxa, Germany) with the V-010 electroporation procedure. Each sample was repeated six times. The transfected cells were cultured for $48 \mathrm{~h}$, and the luciferase activities of DC-SIGN promoter luciferase reporter plasmids and the inner control pRL-TK were detected using the dual luciferase reporter assay kit (Promega) in GloMax96 microplate luminometer (Promega). The relative activity of DC-SIGN promoter was expressed by the ratio of activity between DC-SIGN promoter luciferase reporter plasmids and the inner control pRL-TK.

2.7. Statistics. Every test was repeated three times and data was shown as mean \pm SE. The statistical significance ( $P$ values) of the results was calculated by using SPSS v.13 software. Student's $t$-test was used to compare between two groups 


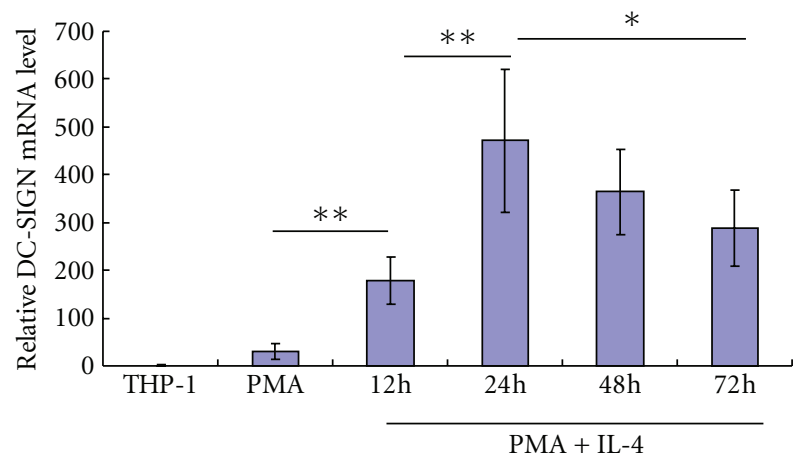

(a)
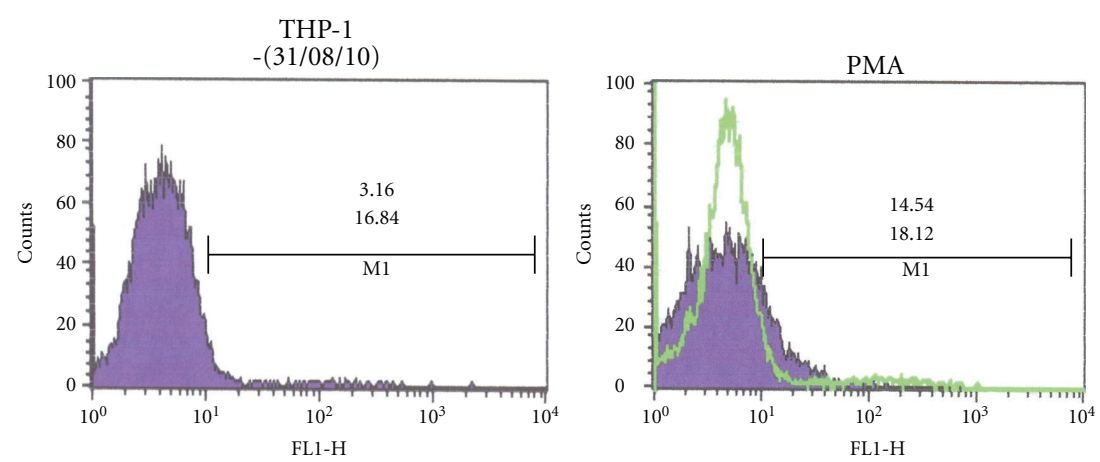

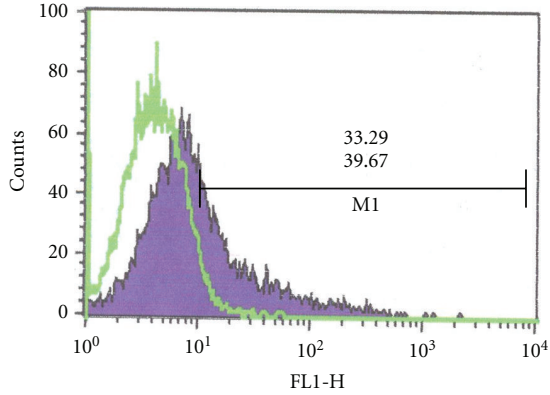

IL-4

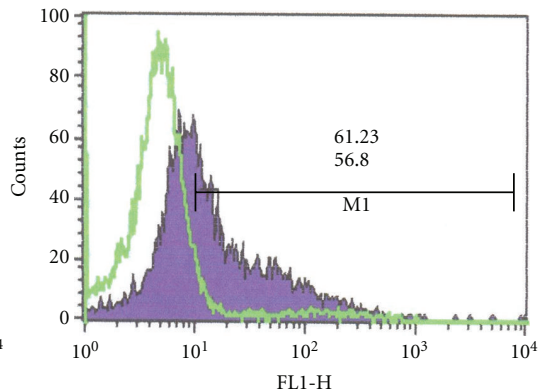

$48 \mathrm{~h}$

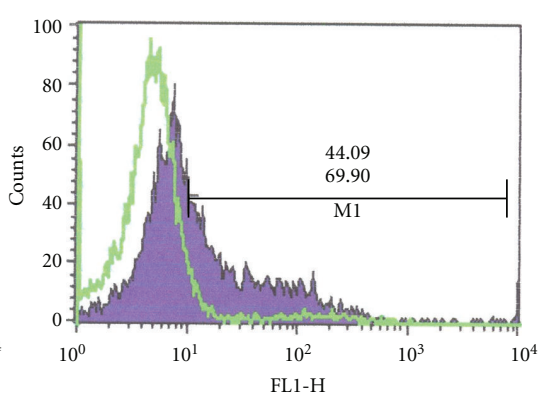

$72 \mathrm{~h}$

(b)

FiguRe 1: IL-4-induced high expression of DC-SIGN on THP-1 cells over time. THP-1 cells were treated with or without PMA for 24 hours, or with PMA for 24 hours and IL-4 for up to 72 hours. (a), the quantitative analysis of the level of DC-SIGN mRNA in differentiated THP-1 cells by PMA and IL-4 by SYBR Green real-time PCR. (b), analysis of induced DC-SIGN expression on surface of differentiated THP-1 cells by flow cytometry. The percentage of positive cells (top number) and mean fluorescence intensity (figure below) are shown. ${ }^{*}$ means $P<0.05 ;{ }^{* *}$ means $P<0.01$.

while one-way ANOVA was used when comparing more than three groups.

\section{Results}

3.1. IL-4 Induced High Expression of DC-SIGN on THP-1 Cells. We determined the DC-SIGN mRNA and expression on untreated, PMA-treated, and PMA plus IL-4-treated THP- 1 cells at different times of differentiation. The results of mRNA testing by real-time quantitative PCR showed that PMA differentiation for 24 hours increased the level of DCSIGN mRNA in THP-1 cells up to 30 folds and induction of IL-4 greatly enhanced the level of DC-SIGN mRNA. The highest level of DC-SIGN mRNA was detected when induced by PMA and IL- 4 for 24 hours, which was $469 \pm 148$ times higher than that of untreated THP- 1 cells $(P<0.01$, see Figure 1(a)).

DC-SIGN is a transmembrane protein. Therefore, we further detected DC-SIGN expression on cell surface by flow cytometry. The results showed that PMA induced a low level of DC-SIGN expression on THP-1 cell surface with the percentage of $14.54 \pm 3.97 \%$ DC-SIGN+ THP-1 cells and the mean fluorescence intensity (MFI) of $18.12 \pm 7.51$. IL-4 greatly enhanced the percentage of DC-SIGN+ THP-1 cells, and the MFI at the same time. The highest expression of DC-SIGN on THP- 1 cells differentiated by PMA plus IL-4, 


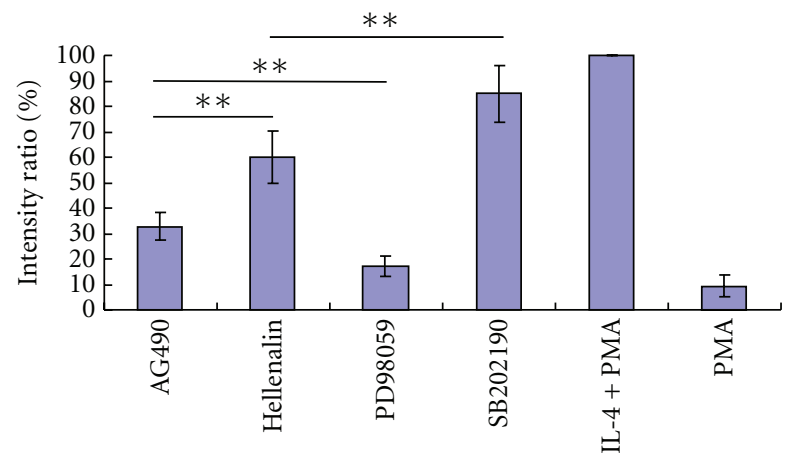

(a)
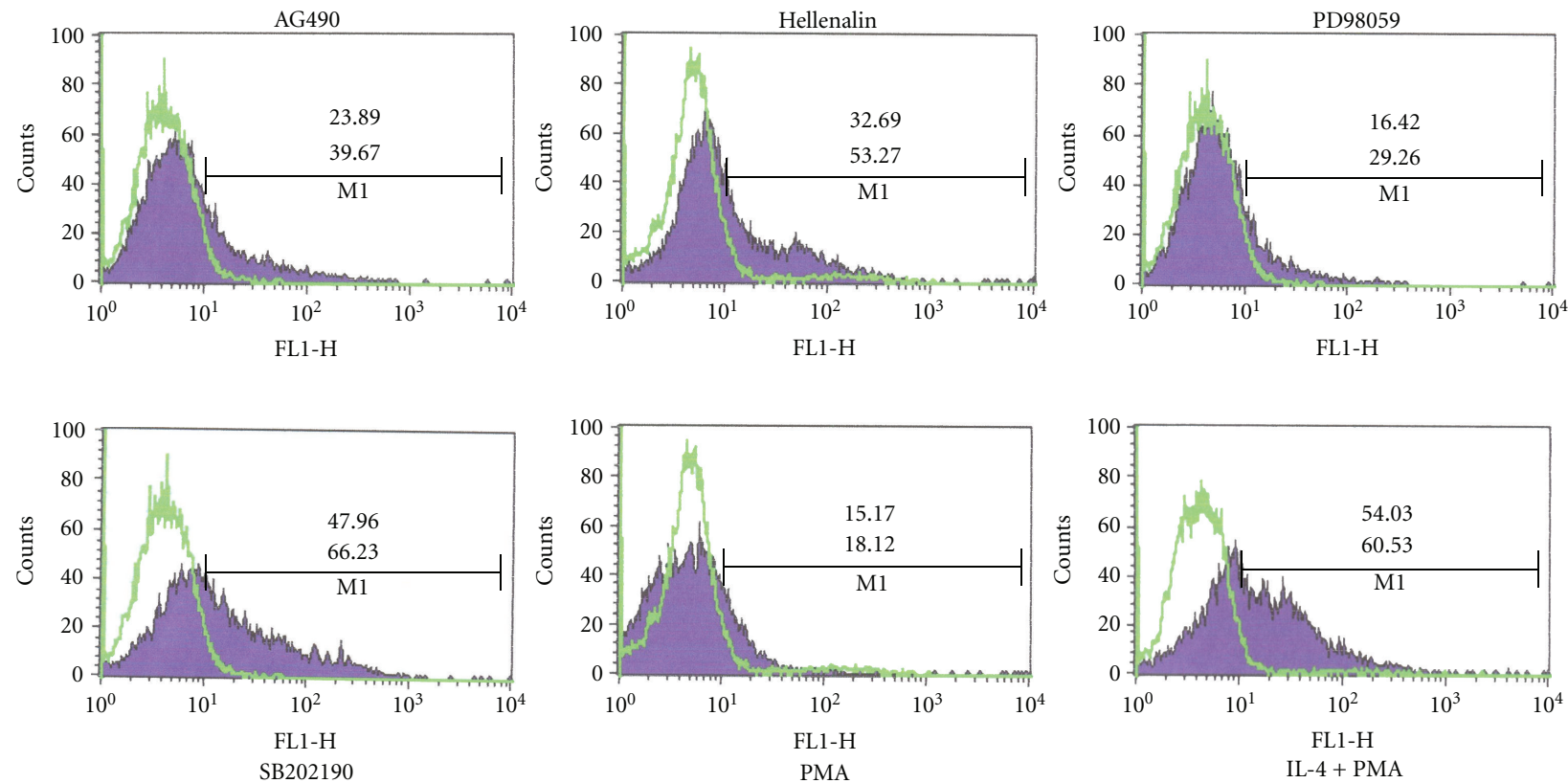

(b)

FIGURE 2: Inhibition of DC-SIGN expression by specific inhibitors of signaling pathways. DC-SIGN expression was detected on THP-1 cells differentiated by PMA, PMA plus IL-4, or PMA plus IL-4 treated with specific inhibitors (Helenalin, AG490, SB202190, and PD98059). (a), The relative levels of DC-SIGN mRNA detected by real-time PCR. The levels of DC-SIGN mRNA in PMA plus IL-4 treated THP-1 cells were valued as 100\%, and used as a reference. * means $P<0.05$; ** means $P<0.01$. (b), DC-SIGN expression on surface of THP- 1 cells detected by flow cytometry. The percentage of positive cells (top number) and mean fluorescence intensity (number below) are shown.

with the percentage of $61.23 \pm 15.21 \%$ DC-SIGN+ THP-1 cells and the MFI of $56.80 \pm 21.35$, was found at 72 hours (24 h for PMA and $48 \mathrm{~h}$ for PMA plus IL-4). We found that the majority of the cells were overactivated and aging after differentiated by PMA plus IL- 4 for 96 hours, and the proportion of dead cells increased. Although the DCSIGN (+) THP-1 ratio was declined, the MFI remained high (Figure 1(b)).

3.2. Intracellular Signaling Pathways Involved in DC-SIGN Expression. The stimulation by IL-4 on IL-4 receptor was mainly transducted through the JAK-STAT and ERK signal pathways [24-27]. In addition, our previous study suggested that the NF- $\kappa \mathrm{B}$ signaling pathway may also be involved in the expression of DC-SIGN [28]. We selected four different alternative pathways (ERK, NF- $\kappa$ B, JAK-STAT, and p38MAPK) as the target signaling pathways, and detected the DC-SIGN expression by blocking the corresponding signaling pathways with specific inhibitors.

Real-time PCR showed that inhibitor of ERK pathway (PD98059) blocked the expression of DC-SIGN mRNA by $83.84 \pm 4.13 \%$, which was the most obvious among the four inhibitors, followed by the inhibitor of JAK-STAT pathway (AG490) which decreased DC-SIGN mRNA by $67.16 \pm$ $5.67 \%$. Blocking of the NF- $\kappa \mathrm{B}$ pathway also decreased DCSIGN mRNA by $40.08 \pm 10.12 \%$. Blocking of DC-SIGN mRNA by inhibitor p38MAPK pathway (SB202190) was not significant (see Figure 2(a)).

We further detected expression of DC-SIGN on THP1 cell membrane using flow cytometry by blocking the signaling pathways with specific inhibitors. DC-SIGN expression was decreased from DC-SIGN+ rate of $54.03 \pm 7.66 \%$ 


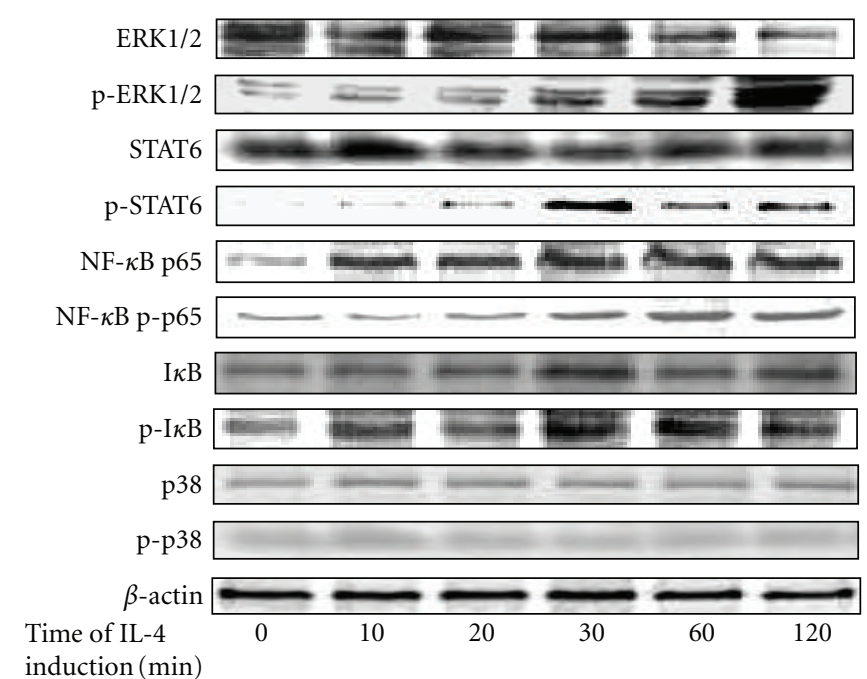

(a)

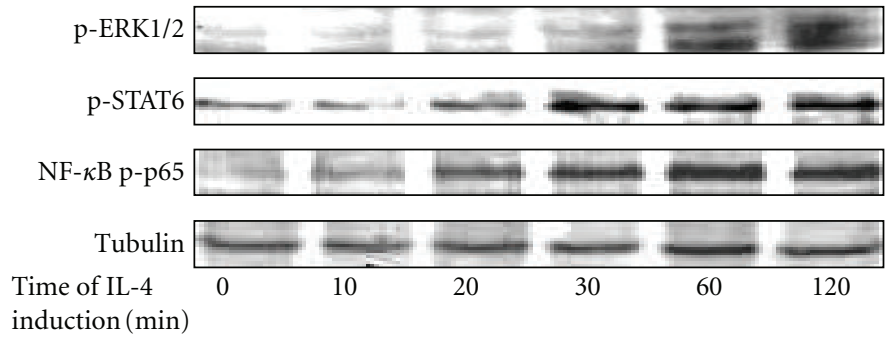

(b)

FIGURE 3: Phosphorylation of kinase and factors over time in the signaling pathways within 120 min in IL-4-induced THP-1 cells. (a), the cytoplasmic levels of protein kinase and phosphorylated kinase in the signaling pathways. (b), the concentration of phosphorylated protein kinase in the nucleus over time. Protein kinase with " $p$ " means phosphorylated protein kinase.

$(\mathrm{MFI}=60.53 \pm 15.42)$ on THP-1 cells induced by PMA+IL-4 to $16.42 \pm 5.88 \%$ (MFI $=29.26 \pm 9.76)$ on differentiated THP1 cells treated by PD98059, close to the PMA-treated THP-1 cells $(15.17 \pm 4.47 \%$, MFI $=18.12 \pm 7.58$. $)$, which mean the nearly total block of IL-4 induction. AG490 decreased DCSIGN expression by $55.8 \%$ with DC-SIGN+ THP-1 cells of $23.89 \pm 5.12 \%(\mathrm{MFI}=39.67 \pm 15.46)$. Hellenalin decreased DC-SIGN expression by $40 \%$ with DC-SIGN+ THP-1 cells of $32.69 \pm 6.69 \%(\mathrm{MFI}=53.27 \pm 18.53)$. Expression of DCSIGN on THP-1 cells treated with SB202190 was almost not reduced (see Figure 2(b)).

3.3. Phosphorylation of Kinase and Factors over Time in the Signaling Pathways. In order to get the direct evidence of activation of the signaling pathways, we examined the phosphorylation of kinase and factors in the signaling pathways. The result of Western Blot test showed that, within the $120 \mathrm{~min}$ after addition of IL-4, the cytoplasmic levels of phosphorylated ERK1/2 of ERK pathway, phosphorylated STAT6 of JAK-STAT pathway, and phosphorylated NF- $\kappa$ Bp65 and $\mathrm{I}-\kappa \mathrm{B}$ of $\mathrm{NF}-\kappa \mathrm{B}$ pathway increased over time from a low concentration to a high concentration, which indicated directly the activation of the three signaling pathways. However, the level of phosphorylated p38 of p38MAPK pathway showed no increase in cytoplasm, indicating the inactivity of the p38MAPK pathway (Figure 3(a)).

We further determined whether the phosphorylated ERK1/2, STAT6 and NF- $\kappa$ Bp65 enter the nucleus to activate DC-SIGN promoter directly or through other nuclear factors. Nuclear proteins were extracted and the phosphorylated kinase was tested by Western Blot. The results showed a similar trend of increase of phosphorylated ERK1/2, STAT6, and NF- $\kappa$ Bp 65 in the nucleus of PMA plus IL-4-induced THP-1 cells within the first $120 \mathrm{~min}$ of IL-4 induction (Figure 3(b)).

3.4. Ets-1 Transcription Factor Binding Site Makes the Main Contribution to the Activity of DC-SIGN Promoter. Liu et al. [29] reported that there are five nuclear transcription factor binding sites (AP-1, SP-1, Ets-1, Lyf-1, and NF- $\kappa \mathrm{B}$ ) in the cis acting elements of DC-SIGN promoter. We studied the role of AP-1 and Ets-1 nuclear transcription factor binding sites in the activity of DC-SIGN promoter, which are the main nuclear factors exploited by ERK signaling pathway, using luciferase reporter system. The sequence of complete DC-SIGN promoter and the mutants with AP-1 or Ets1 binding site deletion were identified by gene sequencing (Figure 4). The results showed that DC-SIGN promoter 
Position: $\quad+331$

$+400$

Complete: TCTGATGCTTTCCACTAGCCTTTTGGATGACAGATCCCTACCCAACTTCCTGTTTCTCTTTCTGTGGGAG

AP-1 mutant: TCTGATGCTTTCCACTAGCCTTTTG- - - - - - - . CCCTACCCAACTTCCTGTTTCTCTTTCTGTGGGAG

Ets-1 mutant: TCTGATGCTTTCCACTAGCCTTTTGGATGACAGATCCCTACCCA- - - - - - - - CTCTTTCTGTGGGAG

Figure 4: Comparison of complete DC-SIGN promoter and the mutants with AP-1 or Ets-1 binding site deletion. The AP-1 binding site at +356 to +365 and Ets- 1 binding site at +375 to +385 of DC-SIGN promoter were deleted.

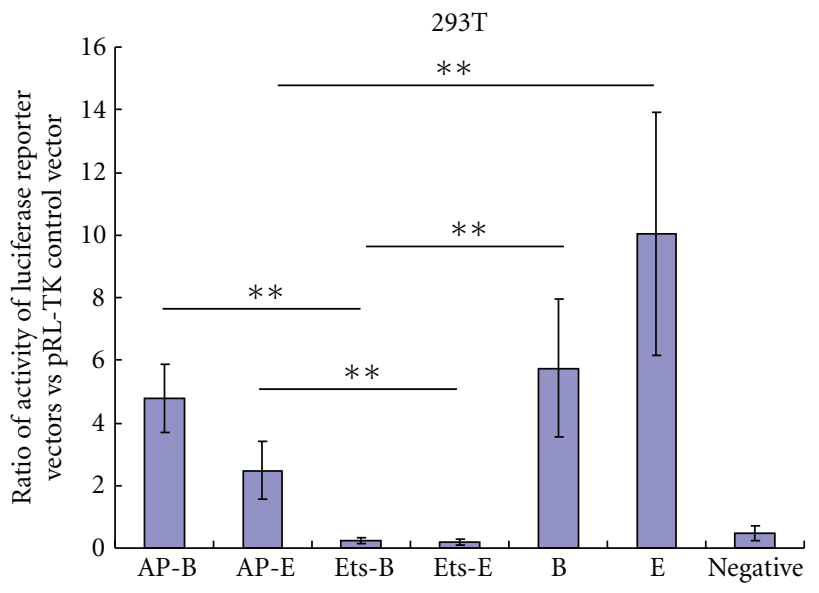

(a)

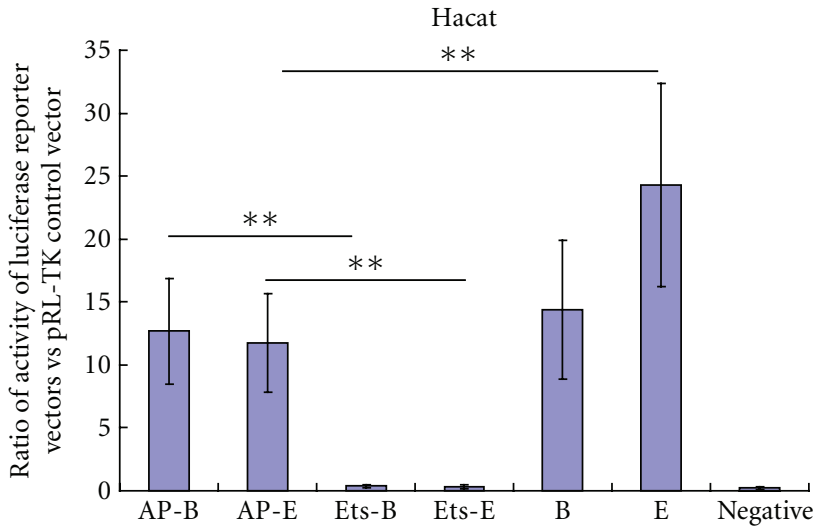

(b)

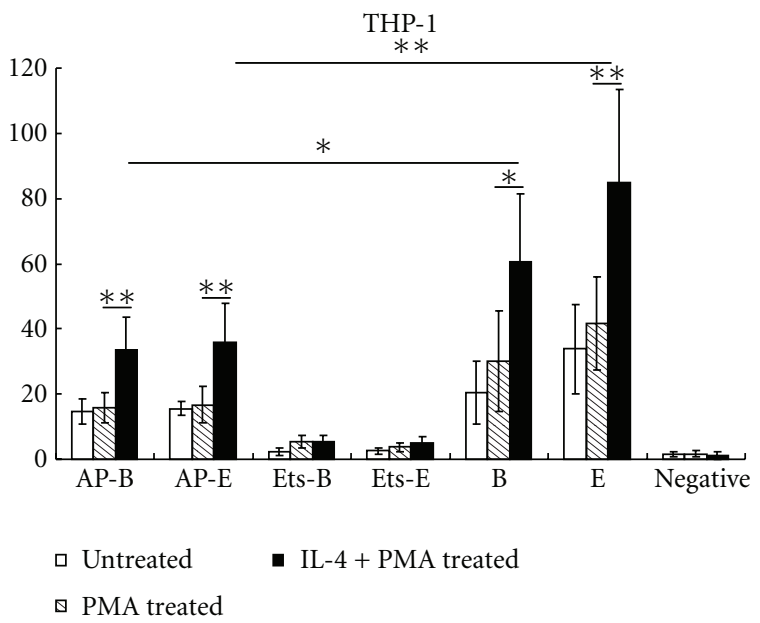

(c)

FIGURE 5: The relative activity of complete DC-SIGN promoters and those without AP-1 or ETS-1 transcription factor binding factors. The activities of luciferase reporter vectors of different DC-SIGN promoters were detected in 293T cells (part a), Hacat cells (part b) and untreated, PMA or PMA+IL-4-treated THP-1 cells (part c). The abbreviation in the figures: AP-B, pGL-3/Basic/DC-SIGN without AP1 transcription factor binding factor; AP-E, pGL-3/Enhancer/DC-SIGN without AP-1 transcription factor binding factor; ETS-B, pGL3/Basic/DC-SIGN without ETS-1 transcription factor binding factor; ETS-E, pGL-3/Enhancer/DC-SIGN without ETS-1 transcription factor binding factor; B, pGL-3/Basic/DC-SIG; E, pGL-3/Enhancer/DC-SIGN; Negative, the internal control plasmid pRL-TK. * means $P<0.05$; ** means $P<0.01$.

activity in THP-1 cells was much higher than in Hacat cells and $293 \mathrm{~T}$ cells. The deletion of Ets-1 nuclear transcription factor binding site had significant impact on the DC-SIGN promoter activity, no matter in Hacat cells, 293T cells, and untreated or PMA-treated or PMA plus IL-4-treated THP-1 cells. The activity of DC-SIGN promoters without Ets-1, no matter the basic or containing enhancers, almost disappeared completely (decreased more than 90\%, $P<0.05$ ). The activity of DC-SIGN promoters without AP-1 decreased partially, from $28.60 \%$ to $47.88 \%$ in different cell lines, which was most obvious in the THP- 1 cell line $(P<0.05$, Figure 5). 


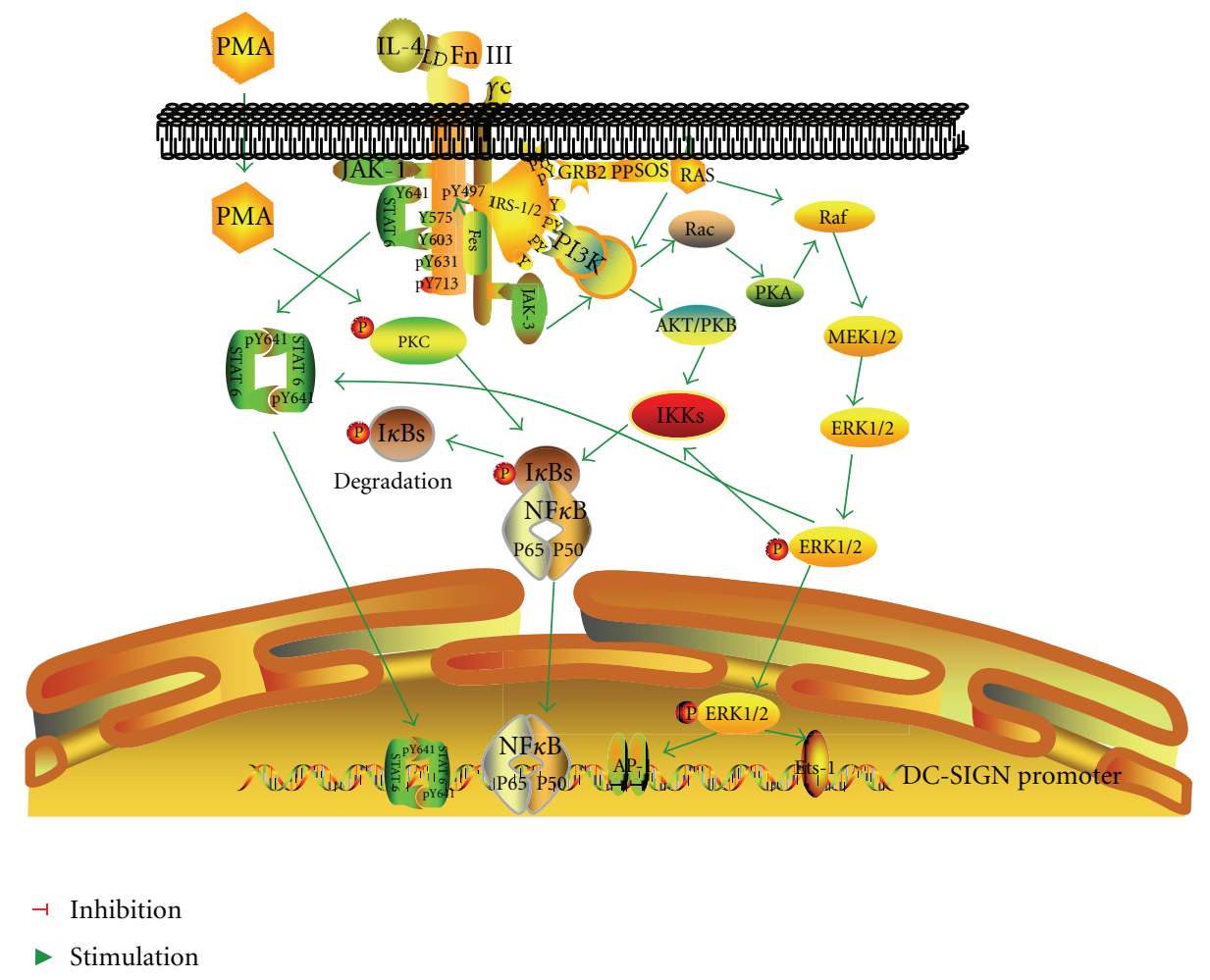

FIGURE 6: Signaling pathways involved in IL-4-induced DC-SIGN expression on THP-1 cells. ERK signaling pathway plays a main role by transcription factor of Ets- 1 to active DC-SIGN promoter. JAK-STAT and NF- $\kappa$ B signaling pathways are also involved, which may be induced by IL-4/IL-4R directly or by the interactions with ERK pathway indirectly.

\section{Discussion}

THP-1 cells can be differentiated into monocytes/macrophages by PMA [30, 31]. Puig-Kröger et al. [32] found that differentiated THP-1 cells can be induced into DCs by IL4 with the expression of DC-SIGN, and PMA stimulation can greatly enhance DC-SIGN expression. Our study further identified IL-4-differentiated THP-1 cells as a good in vitro cell model of DC-SIGN expression. We found that IL-4 can significantly induce high expression of DC-SIGN in both percentage of positive cells and expression density on the cell surface, which demonstrates that DC-SIGN expression is IL4 dependent. The highest expression of DC-SIGN on THP-1 cells differentiated by PMA plus IL- 4 was found at 72 hours (24 h for PMA and $48 \mathrm{~h}$ for PMA plus IL-4), which was different from the results of Puig-Kröger's study with the highest expression at $96 \mathrm{~h}$ ( $24 \mathrm{~h}$ for PMA and $72 \mathrm{~h}$ for PMA plus IL4) [32]. And our study also found that the level of DC-SIGN mRNA was significantly increased, indicating that expression of DC-SIGN is enhanced by IL-4 on the level of mRNA.

The IL-4-initiated signaling pathways that enhance expression of DC-SIGN are still unclear. Researchers have found that expression of many genes in T lymphocytes is induced by IL-4 in an STAT-6-dependent manner [26, 27]. Recent studies have revealed that the JAK 2/3 inhibitor tyrphostin AG-490 can prevent DC-SIGN upregulation on MDDCs and differentiated THP-1 cells, suggesting that STAT6 activation participates in IL-4-induced DC-SIGN expression [14, 32]. IL-4-initiated STAT6 activation is not sufficient for DC-SIGN upregulation to take place, because IL-4 treatment of proliferating THP-1 cells leads to STAT6 activation, but not to DC-SIGN upregulation, thus suggesting the involvement of additional pathways in the IL-4dependent DC-SIGN upregulation in THP-1 cells. Our study reveals that the ERK1/2 inhibitor PD98059 can almost completely inhibit the upregulated DC-SIGN expression induced by IL-4, suggesting that IL-4-induced upregulation of DCSIGN expression is mainly dependent on the ERK MAPK signaling pathway. And the JAK-STAT signaling pathway is also involved in the process for the partial inhibition of IL4-induced DC-SIGN expression by STAT6 inhibitor AG490, which is consistent with the previous studies $[14,32]$. In addition, in ERK MAPK signaling pathway, gene activation is mainly regulated through the transcription factors Ets1 and AP-1, and the forming of a heterodimer of Ets-1 [33]. We further studied the activity of DC-SIGN promoters without Ets-1 or AP-1 transcription factor binding sites and found that the activity of DC-SIGN promoter without Ets-1 transcription factor binding site almost completely disappeared, indicating that the Ets-1 transcription factor binding site plays a key role in the activation of DCSIGN promoter. The deletion of AP-1 transcription factor binding site can make the activity of DC-SIGN promoter decreased partly, the reason of which may be the block of heterodimer of Ets-1 and AP-1 binding to the cis acting elements of DC-SIGN promoter. In addition, we detected the 
phosphorylation of protein kinase in the signaling pathways, and found that the levels of phosphorylated ERK1/2 of ERK pathway and phosphorylated STAT-6 of JAK-STAT pathway gradually increased over time after IL- 4 addition, which provides direct evidence for the activation of the signaling pathways. The increased levels of phosphorylated ERK1/2 and STAT- 6 in the nucleus provide further proof of the activation of the signaling pathways. No increased level of phosphorylated p38 kinase is found in either the cytoplasm or nucleus of differentiated THP-1 cells, indicating that the p38 pathway, which also belongs to the family of MAPK signaling pathways, like the ERK pathway, is not activated by IL-4 in the expression of DC-SIGN.

Another signaling pathway we found involved in IL-4 induced high expression of DC-SIGN on THP- 1 cells is the NF- $\kappa \mathrm{B}$ pathway, of relevance in a variety of inflammation and immunological responses. Our previous study revealed that the deletion of NF- $\kappa \mathrm{B}$ transcription factor binding site allows the activity of DC-SIGN promoter to be reduced by half, and the overexpression of NF- $\kappa \mathrm{B}$ protein can increase the expression of DC-SIGN on THP-1 cells, suggesting that NF- $\kappa \mathrm{B}$ signaling pathway may be involved in the expression of DC-SIGN [28]. Another study revealed that dexamethasone, the inhibitor of NF- $\kappa \mathrm{B}$ signaling pathway, can reduce the expression of DC-SIGN [34]. Combined with the reduced expression of DC-SIGN by inhibiting NF- $\kappa \mathrm{B}$ signaling pathway and the increase of phosphorylated NF- $\kappa B$ in cytoplasm and nucleus of differentiated THP- 1 cells, it can be inferred that the NF- $\kappa \mathrm{B}$ signaling pathway is also involved in the process of upregulated DC-SIGN expression. The NF$\kappa \mathrm{B}$ signaling pathway may be activated by two inducers. One is PMA, which can induce the differentiation of THP- 1 cells through activation of PKC and NF- $\kappa \mathrm{B}$ signaling pathway. The other is IL- 4 , which can directly activate the NF- $\kappa$ B signaling pathway. The synergistic effect of PMA and IL-4 found in other studies, which can highly increase the expression of DC-SIGN on THP- 1 cells $[14,28,32]$, can be attributed to the activation of the NF- $\kappa \mathrm{B}$ signaling pathway.

In summary, our study demonstrates that multiple signaling pathways are involved in the process of IL-4-induced high expression of DC-SIGN on THP-1 cells, in which ERK pathway is the main signaling pathway, and JAK-STAT, and NF- $\kappa$ B signaling pathways are also involved. The JAK-STAT and NF- $\kappa$ B signaling pathways may be activated directly by IL-4/IL-4 receptor, or by the ERK pathway indirectly. Complex interactions may exist between the signaling pathways, forming a regulatory network (see Figure 6). Further studies are needed in the promotion or inhibition effect between the signaling pathways. In addition, the regulatory mechanisms of intracellular signaling pathways vary in different cells, and signaling pathways regulating DC-SIGN expression should be studied in other cells.

\section{Conflict of Interests}

The authors confirm that there are no conflict of interests.

\section{Acknowledgments}

This work was supported by the Special Grant for National Key Technologies R\&D Programme for the 12th Five-Year Plan of China (no. 2012ZX10001-004-005) and National Natural Science Foundation of China (30872241), Beijing.

\section{References}

[1] B. M. Curtis, S. Scharnowske, and A. J. Watson, "Sequence and expression of a membrane-associated C-type lectin that exhibits CD4-independent binding of human immunodeficiency virus envelope glycoprotein gp 120," Proceedings of the National Academy of Sciences of the United States of America, vol. 89, no. 17, pp. 8356-8360, 1992.

[2] T. B. H. Geijtenbeek, R. Torensma, S. J. van Vliet et al., "Identification of DC-SIGN, a novel dendritic cell-specific ICAM-3 receptor that supports primary immune responses," Cell, vol. 100, no. 5, pp. 575-585, 2000.

[3] A. Engering, T. B. H. Geijtenbeek, S. J. van Vliet et al., "The dendritic cell-specific adhesion receptor DC-SIGN internalizes antigen for presentation to T cells," Journal of Immunology, vol. 168, no. 5, pp. 2118-2126, 2002.

[4] K. W. Schjetne, K. M. Thompson, T. Aarvak, B. Fleckenstein, L. M. Sollid, and B. Bogen, "A mouse $\mathrm{C} \kappa$-specific T cell clone indicates that DC-SIGN is an efficient target for antibodymediated delivery of $\mathrm{T}$ cell epitopes for MHC class II presentation," International Immunology, vol. 14, no. 12, pp. 1423-1430, 2002.

[5] T. B. H. Geijtenbeek, D. J. E. B. Krooshoop, D. A. Bleijs et al., "DC-SIGN-1CAM-2 interaction mediates dendritic cell trafficking," Nature Immunology, vol. 1, no. 4, pp. 353-357, 2000.

[6] A. Cambi and C. G. Figdor, "Dual function of C-type lectinlike receptors in the immune system," Current Opinion in Cell Biology, vol. 15, no. 5, pp. 539-546, 2003.

[7] B. J. Appelmelk, I. van Die, S. J. van Vliet, C. M. J. E. Vandenbroucke-Grauls, T. B. H. Geijtenbeek, and Y. Van Kooyk, "Cutting edge: carbohydrate profiling identifies new pathogens that interact with dendritic cell-specific ICAM-3grabbing nonintegrin on dendritic cells," Journal of Immunology, vol. 170, no. 4, pp. 1635-1639, 2003.

[8] T. B. H. Geijtenbeek, D. S. Kwon, R. Torensma et al., "DCSIGN, a dendritic cell-specific HIV-1-binding protein that enhances trans-infection of T cells," Cell, vol. 100, no. 5, pp. 587-597, 2000.

[9] S. Pöhlmann, F. Baribaud, B. Lee et al., "DC-SIGN interactions with human immunodeficiency virus type 1 and 2 and simian immunodeficiency virus," Journal of Virology, vol. 75, no. 10, pp. 4664-4672, 2001.

[10] E. J. Soilleux, L. S. Morris, G. Leslie et al., "Constitutive and induced expression of DC-SIGN on dendritic cell and macrophage subpopulations in situ and in vitro," Journal of Leukocyte Biology, vol. 71, no. 3, pp. 445-457, 2002.

[11] A. Engering, S. J. van Vliet, K. Hebeda et al., "Dynamic populations of dendritic cell-specific ICAM-3 grabbing nonintegrin-positive immature dendritic cells and liver/lymph nodespecific ICAM-3 grabbing nonintegrin-positive endothelial cells in the outer zones of the paracortex of human lymph nodes," American Journal of Pathology, vol. 164, no. 5, pp. 1587-1595, 2004.

[12] A. Engering, S. J. van Vliet, T. B. H. Geijtenbeek, and Y. Van Kooyk, "Subset of DC-SIGN" dendritic cells in human blood 
transmits HIV-1 to T lymphocytes," Blood, vol. 100, no. 5, pp. 1780-1786, 2002.

[13] C. Trumpfheller, C. G. Park, J. Finke, R. M. Steinman, and A. Granelli-Piperno, "Cell type-dependent retention and transmission of HIV-1 by DC-SIGN," International Immunology, vol. 15, no. 2, pp. 289-298, 2003.

[14] M. Relloso, A. Puig-Kröger, O. Muñiz Pello et al., "DCSIGN (CD209) expression is IL-4 dependent and is negatively regulated by IFN, TGF- $\beta$, and anti-inflammatory agents," Journal of Immunology, vol. 168, no. 6, pp. 2634-2643, 2002.

[15] R. R. Singh, "IL-4 and many roads to lupuslike autoimmunity," Clinical Immunology, vol. 108, no. 2, pp. 73-79, 2003.

[16] M. Miyaji, R. L. Kortum, R. Surana et al., "Genetic evidence for the role of Erk activation in a lymphoproliferative disease of mice," Proceedings of the National Academy of Sciences of the United States of America, vol. 106, no. 34, pp. 14502-14507, 2009.

[17] F. Sallusto and A. Lanzavecchia, "Efficient presentation of soluble antigen by cultured human dendritic cells is maintained by granulocyte/macrophage colony-stimulating factor plus interleukin 4 and downregulated by tumor necrosis factor $\alpha$, Journal of Experimental Medicine, vol. 179, no. 4, pp. 11091118, 1994.

[18] N. Romani, D. Reider, M. Heuer et al., "Generation of mature dendritic cells from human blood An improved method with special regard to clinical applicability," Journal of Immunological Methods, vol. 196, no. 2, pp. 137-151, 1996.

[19] S. Gordon, "Alternative activation of macrophages," Nature Reviews Immunology, vol. 3, no. 1, pp. 23-35, 2003.

[20] E. Vey, J. H. Zhang, and J. M. Dayer, "IFN- $\gamma$ and 1,25(OH)2D3 induce on THP- 1 cells distinct patterns of cell surface antigen expression, cytokine production, and responsiveness to contact with activated T cells," Journal of Immunology, vol. 149, no. 6, pp. 2040-2046, 1992.

[21] C. Bombara and R. A. Ignotz, "TGF-beta inhibits proliferation of and promotes differentiation of human promonocytic leukemia cells," Journal of Cellular Physiology, vol. 153, no. 1, pp. 30-37, 1992.

[22] J. L. Wood and A. Graham, "Reduction of transition metals by human (THP-1) monocytes is enhanced by activators of protein kinase C," Free Radical Research, vol. 31, no. 5, pp. 367379, 1999.

[23] J. Li, X. Shao, L. Wu et al., "Honokiol: an effective inhibitor of tumor necrosis factor- $\alpha$-induced up-regulation of inflammatory cytokine and chemokine production in human synovial fibroblasts," Acta Biochimica et Biophysica Sinica, vol. 43, no. 5, pp. 380-386, 2011.

[24] R. K. Bhogal and C. A. Bona, "Regulatory effect of extracellular signal-regulated kinases (ERK) on type I collagen synthesis in human dermal fibroblasts stimulated by IL-4 and IL-13," International Reviews of Immunology, vol. 27, no. 6, pp. 472496, 2008.

[25] L. H. Wei, Y. Yang, G. Wu, and L. J. Ignarro, "IL-4 and IL13 upregulate ornithine decarboxylase expression by PI3K and MAP kinase pathways in vascular smooth muscle cells," American Journal of Physiology, vol. 294, no. 5, pp. C1198C1205, 2008.

[26] E. Forbes, N. Van Panhuys, B. Min, and G. Le Gros, "Differential requirements for IL-4/STAT6 signalling in CD4 Tcell fate determination and Th2-immune effector responses," Immunology and Cell Biology, vol. 88, no. 3, pp. 240-243, 2010.

[27] K. Takeda, T. Kishimoto, and S. Akira, "STAT6: its role in interleukin 4-mediated biological functions," Journal of Molecular Medicine, vol. 75, no. 5, pp. 317-326, 1997.
[28] L. J. Xu, X. C. Chang, H. P. Yao, and N. P. Wu, "NF- $\kappa$ Bp50 is associated with DC-SIGN expression induced by IL-4 in THP1 cells," Progress in Biochemistry and Biophysics, vol. 35, no. 1, pp. 50-55, 2008.

[29] H. Liu, W. Yu, L. Y. Liou, and A. P. Rice, "Isolation and characterization of the human DC-SIGN and DC-SIGNR promoters," Gene, vol. 313, no. 1-2, pp. 149-159, 2003.

[30] H. Schwende, E. Fitzke, P. Ambs, and P. Dieter, "Differences in the state of differentiation of THP-1 cells induced by phorbol ester and 1,25-dihydroxyvitamin D3," Journal of Leukocyte Biology, vol. 59, no. 4, pp. 555-561, 1996.

[31] Y. Wang, J. J. Zhang, W. Dai, and J. W. Pike, "Production of granulocyte colony-stimulating factor by THP-1 cells in response to retinoic acid and phorbol ester is mediated through the autocrine production of interleukin-1," Biochemical and Biophysical Research Communications, vol. 225, no. 2, pp. 639-646, 1996.

[32] A. Puig-Kröger, D. Serrano-Gómez, E. Caparrós et al., "Regulated expression of the pathogen receptor dendritic cellspecific intercellular adhesion molecule 3 (ICAM-3)-grabbing nonintegrin in THP-1 human leukemic cells, monocytes, and macrophages," The Journal of Biological Chemistry, vol. 279, no. 24, pp. 25680-25688, 2004.

[33] E. P. Newberry, D. Willis, T. Latifi, J. M. Boudreaux, and D. A. Towler, "Fibroblast growth factor receptor signaling activates the human interstitial collagenase promoter via the bipartite Ets-AP1 element," Molecular Endocrinology, vol. 11, no. 8, pp. 1129-1144, 1997.

[34] S. J. van Vliet, E. van Liempt, T. B. H. Geijtenbeek, and Y. van Kooyk, "Differential regulation of C-type lectin expression on tolerogenic dendritic cell subsets," Immunobiology, vol. 211, no. 6-8, pp. 577-585, 2006. 


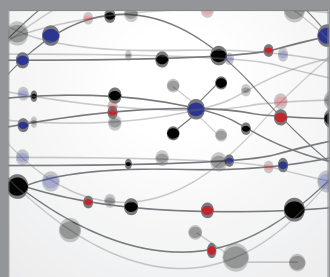

The Scientific World Journal
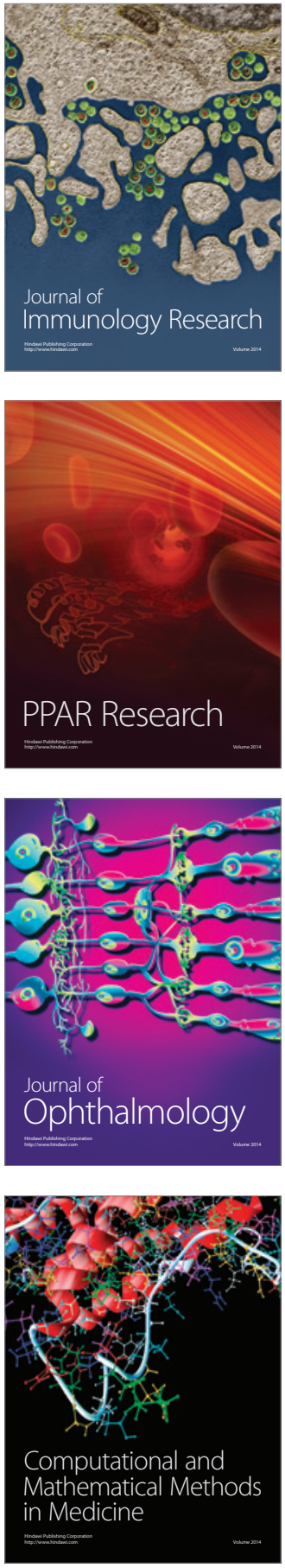

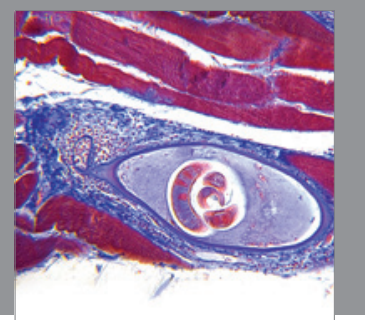

Gastroenterology

Research and Practice
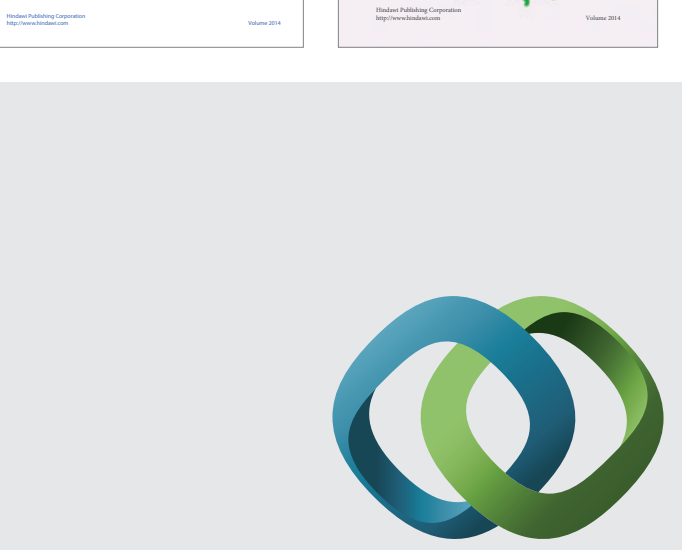

\section{Hindawi}

Submit your manuscripts at

http://www.hindawi.com
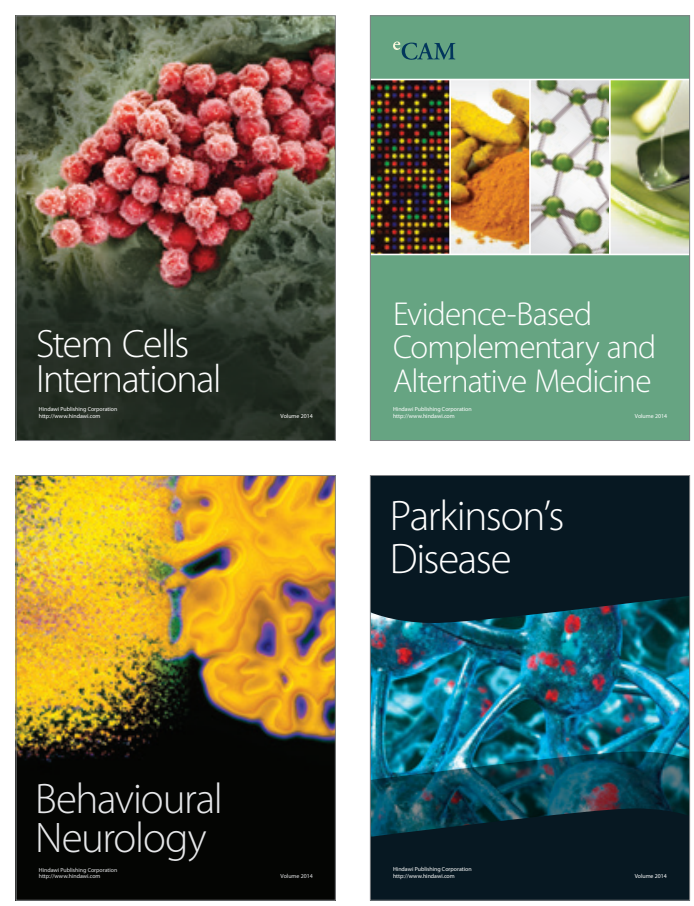

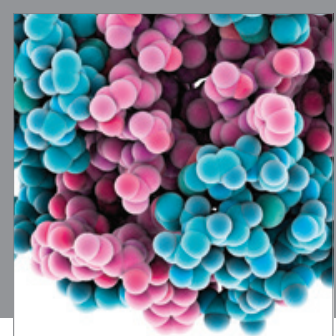

Journal of
Diabetes Research

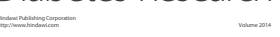

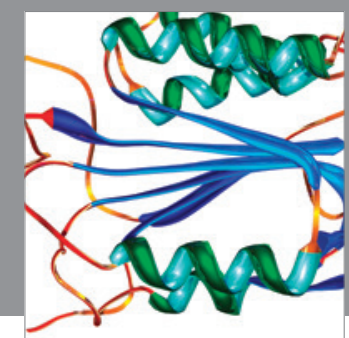

Disease Markers
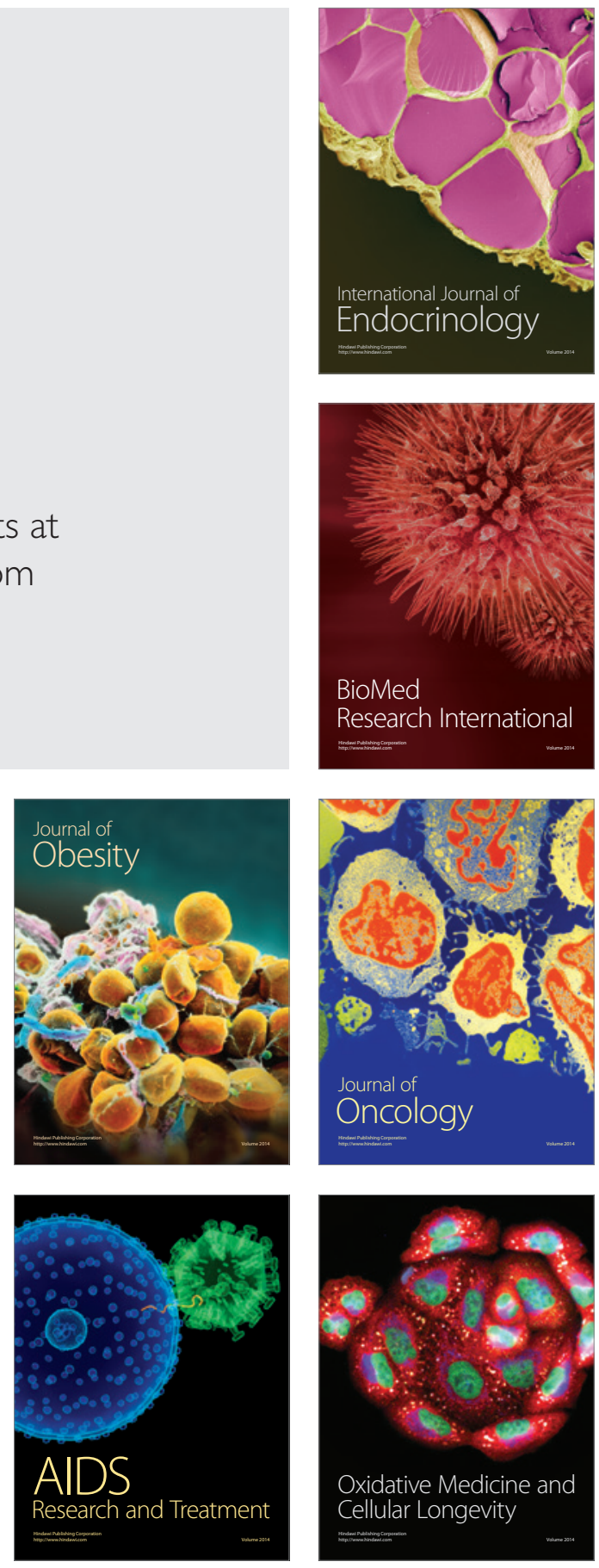